\title{
Progressive gray matter changes in patients with congenital central hypoventilation syndrome
}

\author{
Rajesh Kumar' ${ }^{1}$, Marlyn S. Woo², Paul M. Macey², Mary A. Woo² and Ronald M. Harper ${ }^{1}$
}

INTRODUCTION: Patients with congenital central hypoventilation syndrome (CCHS) show brain injury in areas that control chemosensory, autonomic, motor, cognitive, and emotion functions, which are deficient in the condition. Many of these abnormal characteristics are present from the neonatal period; however, it is unclear whether tissue injury underlying the characteristics progressively worsens with time. We hypothesized that several brain areas in subjects with CCHS would show increased gray matter volume loss over time.

METHODS: We collected high-resolution T1-weighted images twice (4 years apart) from seven subjects with CCHS (age at first study, $16.1 \pm 2.7$ years; four males) and three control subjects (15.9 \pm 2.1 years; three males) using a 3.0-Tesla magnetic resonance imaging (MRI) scanner, and evaluated regional gray matter volume changes with voxel-based morphometry (VBM) procedures.

RESULTS: Multiple brain sites in CCHS, including frontal, prefrontal, insular, and cingulate cortices; caudate nuclei and putamen; ventral temporal and parietal cortices; and cerebellar cortices showed significantly reduced gray matter volume over time. Only limited brain areas, including sensory, temporal, and medullary regions, emerged with increased gray matter at the later age.

DISCUSSION: Patients with CCHS show reduced gray matter volume with age progression in autonomic, respiratory, and cognitive regulatory areas, an outcome that may contribute to deterioration of functions found in the syndrome with increasing age.

C ongenital central hypoventilation syndrome (CCHS), a genetic condition associated with PHOX2B mutations (1), is characterized by reduced sensitivity to $\mathrm{CO}_{2}$ and $\mathrm{O}_{2}$, diminished drive to breath during sleep, and multiple abnormal physiological, motor, and neuropsychological characteristics (2-4). The syndrome is accompanied by neural injury in both gray and white matter regions, as assessed by magnetic resonance imaging (MRI)-based T2-relaxometery, manual volumetric, three-dimensional surface morphometry, and diffusion tensor imaging procedures (5-8), in regions that are implicated in autonomic, physiological, motor, and neuropsychological regulation. The injuries may have arisen from developmental consequences of the $\mathrm{PHOX} 2 \mathrm{~B}$ gene, mutations of which appear to underlie the syndrome (1). However, affected children are also exposed to intermittent hypoxia, an outcome developing from inadequate ventilatory support, especially during sleep, but occasionally during the day in periods of elevated temperature or inactivity. Such hypoxic exposure has the potential to induce or aggravate neural injury (9-12). In addition, impaired perfusion resulting from PHOX2B influences on autonomic development may also contribute to injury progression. It is unclear whether such brain injury increases with time in patients with CCHS.

Several quantitative MRI procedures, including T2relaxometry and diffusion tensor imaging methods, can be used to assess tissue changes over time (13). Both procedures require consistent MRI data-acquisition parameters to allow comparison of pathological changes, and for longitudinal studies, because with scanner upgrades and other issues, scanning parameters may change with time. However, high-resolution T1-weighted images, together with voxel-based morphometry (VBM) analytical procedures, can be used to assess gray matter changes across the brain over time. Because VBM procedures involve partitioning gray from white matter and cerebrospinal fluid tissue types and comparing regional gray matter voxelby-voxel across the brain, slightly altered data-acquisition parameters can be expected not to influence findings for longitudinal assessment or data collected from multiple scanners (14). The techniques have been used to assess gray matter changes in longitudinal and cross-sectional studies $(14,15)$, and may be useful for evaluating gray matter changes with time in subjects with CCHS.

Gray matter tissue injury can be reflected as volume loss in both adult and pediatric conditions. Regional gray matter volume increases with development in the early stages of life in many brain areas and significantly reorganizes during adolescence (16). However, gray matter volume declines with time in adulthood due to normal aging processes (17). Disease-related gray matter volume loss can be assessed only after accounting for normal age-related volume changes in subjects with CCHS.

Our aim was to examine the progression of gray matter injury across the brain in patients with CCHS with VBM 


\section{Articles $\mid$ Kumar etal.}

procedures using high-resolution T1-weighted images. Because of the substantial potential for hypoxic exposure during daily life of children with CCHS, we hypothesized that multiple brain areas would show increased gray matter injury over time.

\section{RESULTS}

Multiple brain areas in control subjects showed reduced gray matter volume with development. Brain sites that emerged with reduced gray matter volume in controls at the later scans over the first scans included both anterior insulae (Figure 1a); bilateral genu extending to anterior cingulate (Figure $\mathbf{1 b}, \mathbf{e}$ ) and ventral medial prefrontal cortices (Figure 1h); mid-cingulate (Figure 1g), bilateral frontal (Figure 1i), parietal (Figure 1f), and temporal operculum (Figure 1d); prefrontal and frontomedial cortices (Figure 1c,j,k); and right caudal cerebellar cortex (Figure 11). A few regions showed increased gray matter volume at the later scan over the earlier scans in control subjects; these included the left rostral cerebellar and right midline occipital cortices.

Several brain regions showed reduced gray matter volume in subjects with CCHS, corrected for normal age-related changes, at the second time point over the initial scans. Brain regions that showed reduced gray matter in CCHS at the later age included the bilateral ventral medial prefrontal (Figure 2a), dorsal prefrontal (Figure 2b), and fronto-medial (Figure 2d) cortices; anterior insula (Figure 2e); caudate nuclei and putamen (Figure 2f,h); genu (Figure 2i); anterior (Figure 2c), mid- (Figure 2j), and posterior cingulate cortices (Figure 2k); ventral temporal (Figure 2m), occipital (Figure 2g,n), and parietal cortices (Figure 21); and bilateral cerebellar cortices (Figure 2o). Only a few sites in subjects with CCHS showed increased gray matter volume over time; these areas included a bilateral region of the dorsal parietal sensory cortex bordering the motor cortices (Figure 3a), as well as temporal (Figure 3b) and dorsal medullary regions (Figure 3c).

\section{DISCUSSION}

\section{Overview}

We investigated the progressive gray matter volume changes across the brain in patients with CCHS, controlling for normal developmental-related changes. Multiple brain regions showed reduced gray matter volume with time in subjects with CCHS in autonomic, mood, motor, and cognitive regulatory areas, which may contribute to deterioration of those regulatory functions over time in the condition. The pathological processes that contribute to such increased injury are unknown, but may include hypoxic processes frequently encountered in subjects with CCHS or sustained perfusion issues resulting from the vascular consequences of the condition.

\section{Gray Matter Changes With Age}

In healthy control subjects, gray matter volume increases at an early stage of life, due to increase in neurons and glia, and volume begins to decline from puberty, a consequence of gray matter maturation and increase in neuronal density (16). Such neuroanatomic changes are region specific and appear with variable maturation patterns in pediatric subjects (16). In adult stages of life, with normal aging, neuronal and other cell loss over time leads to gray matter volume reduction with age (15). Other MRI measures, including T2-relaxation values and diffusion tensor imaging-based indices, also indicate a similar pattern of tissue changes in gray, as well as white matter regions in adult and pediatric control subjects (18-20).

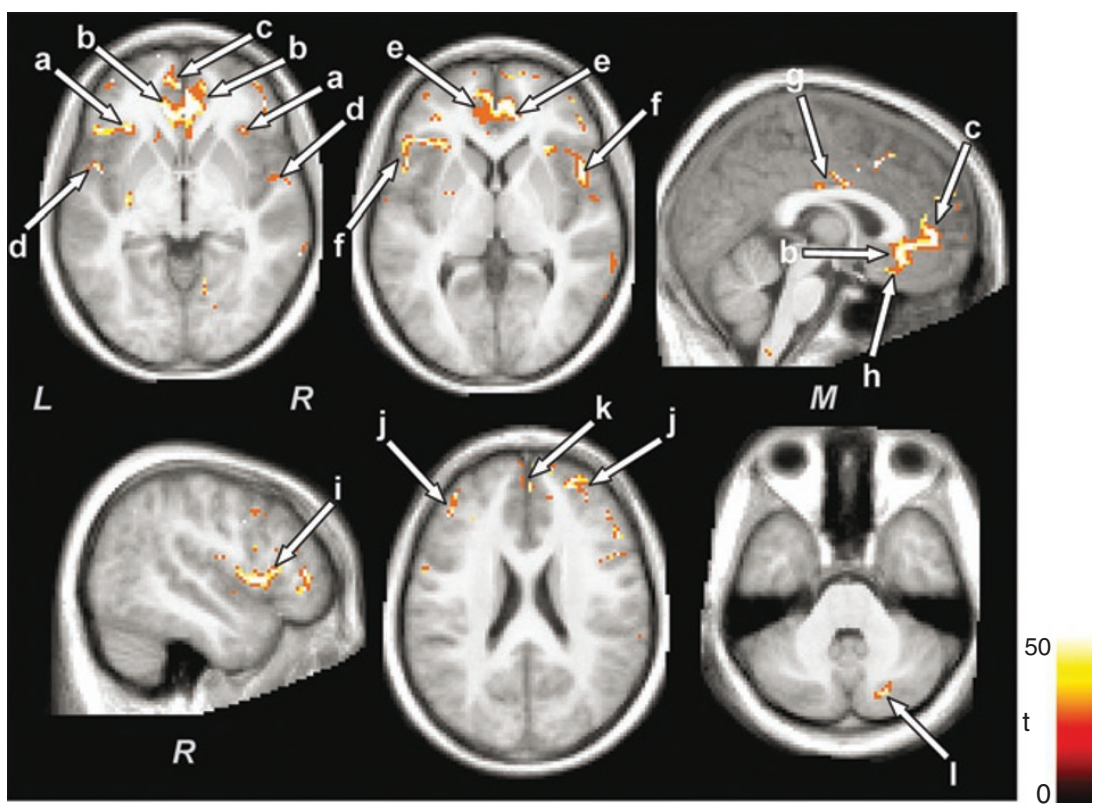

Figure 1. Brain sites with decreased gray matter volume in control subjects with age. Decreased gray matter volume with time emerged in the (a) insula; (b, e, g) cingulate cortices; (c, h, j, k) prefrontal and frontal cortices; (i) frontal, (f) parietal, and (d) temporal operculum; and (I) cerebellum. All brain images are displayed in neurological convention, and color scale represents $t$-statistic values. L, left; M, middle; $R$, right. 


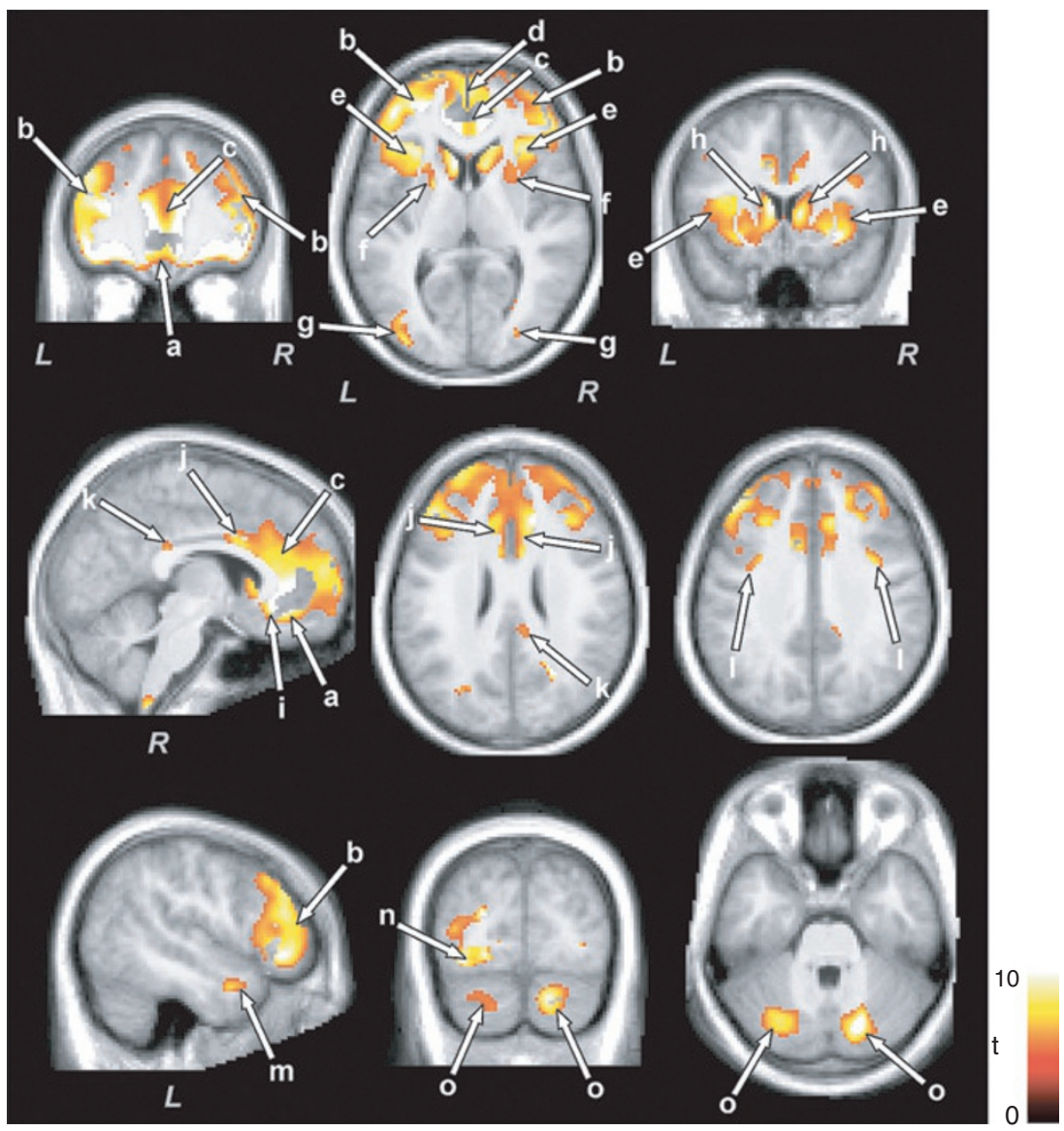

Figure 2. Brain areas with injury progression with time in subjects with congenital central hypoventilation syndrome. Brain regions that showed injury progression included the (a, b, d) prefrontal and frontal cortices; (e) insular regions; $(\mathrm{f}, \mathrm{h})$ caudate nuclei and putamen; (c, i-k) cingulate cortices; (m) temporal, $(g, n)$ occipital, and (l) parietal cortices; and (o) cerebellar cortex. All brain images are displayed in neurological convention, and color scale represents $t$-statistic values. $L$, left; $R$, right.

Many sites in subjects with CCHS showed reduced gray matter volume, even after controlling for normal age-related volume changes. Such significant reductions in gray matter volume indicate progression of tissue injury over time that may result from hypoxic mechanisms commonly encountered in the condition. A few brain regions in subjects with CCHS emerged with increased gray matter volume over time, which possibly results from delayed developmental changes in the condition.

\section{Reduced Gray Matter Volume in Autonomic Regulatory Areas}

Brain sites that play significant roles in autonomic regulation include insular, hypothalamic, ventral medullary, and cerebellar regions (21-25). Most of these areas, including bilateral insular and cerebellar sites, showed increased injury with time in subjects with CCHS. The augmented damage may contribute to worsening autonomic functions in the syndrome, possibly furthering conditions that lead to the reduced lifespan in affected children.

Cerebellar structures, including the cortices, play major roles in blood pressure regulation, especially in coordination of blood pressure changes with body motion or dampening of lows and highs of blood pressure $(21,22)$. To our knowledge, no evidence yet exists that postural blood pressure issues worsen with development in CCHS, but such an issue could readily be examined.

The autonomic roles of the right insular cortex are principally related to sympathetic regulation, and those of the left side are principally related to parasympathetic activity $(26,27)$. Stimulation of the right anterior insula in humans greatly diminishes the baroreflex, whereas posterior insular stimulation elicits cardiac arrhythmia (23). Stroke-related injury of the right insula is followed by a high incidence of myocardial infarction (28), possibly from high sympathetic action related to the right insular damage.

Subjects with CCHS show a range of aberrant cardiovascular issues, including reduced heart rate variability, decreased nocturnal "dipping" of blood pressure, and a propensity for potentially fatal cardiac arrhythmia (29-31); subjects also have a limited lifespan, with sudden death common in the condition. At least some of the sudden deaths apparently result from cardiovascular irregularities in the syndrome (32). Positive associations occur between cardiac disturbances and CCHS severity; with some severities, cardiac deficits appear to be progressive as well (32). Altered autonomic tone resulting from progressive injury in insular and other autonomic areas may contribute to the worsening of the arrhythmia incidence. 


\section{Articles $\mid$ Kumar etal.}

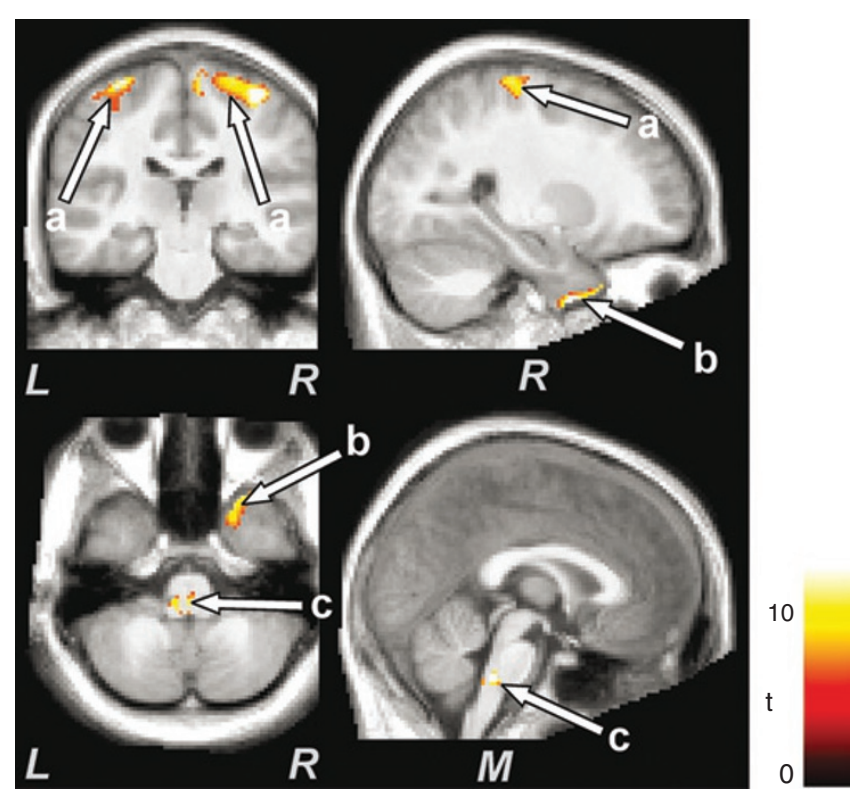

Figure 3. Brain regions with increased gray matter volume over time in subjects with congenital central hypoventilation syndrome. Brain structures that showed increased gray matter volume included (a) primary sensory/motor cortex and (b) temporal and (c) medullary regions. All brain images are displayed in neurological convention, and color scale represents $t$-statistic values. $L$, left; $M$, middle; $R$, right.

\section{Gray Matter Volume Changes in Cognitive, Motor, and Mood} Regulatory Sites

Multiple brain regions in subjects with CCHS showed reduced gray matter volume over time in motor, mood, and cognitive regulatory regions. These sites included caudate, cingulate, prefrontal, frontal, and temporal areas. The caudate nuclei are implicated in motor and cognitive behaviors, including learning, verbal fluency, attention, short- and long-term memory, mental flexibility, and motivation (33-35), and prefrontal and frontal cortices are involved in executive function $(36,37)$. Although prefrontal and frontal cortices also showed increased volume loss in CCHS over time, extensive injury in the caudate nuclei, which project to the frontal and prefrontal cortices, may contribute to the underlying executive dysfunction (38).

The progression of injury in regions serving motivation, as well as in frontal cortices for judgment and behaviors based on comprehension of consequences, is a particular concern, as such neural injury likely contributes to the frequent anecdotal reports of children with CCHS engaging in high-risk behaviors. Some of these behaviors are life threatening, e.g., underwater breath-holding competitions, alcohol consumption, and some lead to carelessness in nocturnal ventilatory use, which places the individual at grave risk, certainly contributing to the short lifespan in these individuals.

Along with autonomic deficits, subjects with CCHS show a variety of motor deficits in addition to the reduced drive to the breathing musculature during sleep (2). These deficits include unilateral smiling following a joke (despite ability to voluntarily smile bilaterally), and eye movement issues (39). Other deficits in CCHS include learning, working memory, attention, and social interaction $(3,4)$. These deficits in motor function and cognition aspects, including learning and memory and executive function in CCHS may result from injury in caudate, frontal, and prefrontal cortices $(33,34,36)$. However, we lack evidence as to whether these functional deficits progressively worsen with age in CCHS.

\section{Potential Mechanisms of Gray Matter Injury}

Multiple pathological mechanisms in CCHS may have contributed to gray matter volume loss over time. As the major signs, including failed breathing drive during sleep and severe autonomic symptoms, appear early in life, brain injury incurred by PHOX2B mutations in early stages may contribute to these deficient functions. However, the autonomic sequelae in subjects with CCHS also alter the cerebral vasculature (40), and those changes may modify perfusion to brain structures, with obvious deleterious consequences, in addition to the repeated hypoxia exposure from loss of ventilatory drive during sleep with failed ventilatory support. We believe that much of the injury to medullary areas in CCHS, especially injury to the raphe system (7); the damage to the locus coeruleus, one structure where PHOX2B is localized; and possibly the hypothalamic damage, due to unique pattern of injury (8), result from initial PHOX2B mutations, with hippocampal, possibly cerebellar, and cortical damage found here resulting from perfusion and hypoxia issues.

\section{Limitations}

Some limitations of the study should be acknowledged, including use of different MRI scanning parameters for data acquisition, which may influence the findings, and the limited number of subjects, including only male controls. The relative neuroprotection offered by the female sex may significantly reduce hypoxic or other injury in female adolescents. The MRI scanner software was upgraded repeatedly over time, disallowing identical scanning parameters, resulting in slightly different scanning parameters to acquire the high-resolution T1-weighted images. However, the analytical procedures used here, VBM, require partitioning gray matter from other tissue types, and comparison of whole-brain regional gray matter changes; small differences in scanning parameters should not drastically influence findings. Such procedures, with variable scanning parameters, have been used reliably to assess tissue changes using data collected from multiple scanners (14). The rare nature of the CCHS condition restricted follow-up to a limited number of CCHS subjects. Moreover, relocation issues limited follow-up of control subjects.

\section{Conclusions}

Patients with CCHS show progressive gray matter volume loss, after partitioning for normal age-related tissue changes, in several brain regions that control autonomic, mood, motor, and cognitive functions. Only a few areas emerge with increased gray matter over time in subjects with CCHS. Such progressive gray matter injury in autonomic, motor, and cognitive regulatory regions may contribute to worsening of essential functions found 
in the condition, including protection against life-threatening risk behaviors and autonomic characteristics that enhance protection against fatal arrhythmia. The pathological mechanisms contributing to progression of injury with age are unknown, but likely include hypoxic processes that accompany the syndrome.

\section{METHODS}

\section{Subjects}

We studied seven subjects with CCHS and three control subjects twice, $\sim 4$ years apart. The demographic data and other characteristics of subjects with CCHS and control subjects are summarized in Table 1. The diagnosis of CCHS was based on the American Thoracic Society criteria (1999; ref. 2), and subjects with CCHS were recruited through the CCHS family network (http://www.cchsnetwork.org). All subjects with CCHS had symptoms of moderate severity, with requirement of ventilator support only during the night; subjects requiring ventilatory support during the day were not included. Subjects with CCHS with other conditions that may induce brain injury, including cardiovascular or neurological conditions, or with diagnosed Hirschsprung's disease, which may introduce malnutrition (increased risk for neural injury) through malabsorption issues, were excluded as well. All control subjects were healthy, without any history of neurological or other issues that may induce brain injury, and were recruited through advertisements at the university campus and surrounding area.

Brain imaging studies of subjects with CCHS and control subjects were performed without any anesthesia or sedatives, and subjects were provided rest from the scanner if required. The study protocol was approved by the institutional review board of the University of California at Los Angeles, and subjects with CCHS and control subjects or their parents/caretakers provided informed written consent before the study.

\section{MRI}

Brain imaging studies were performed using a 3.0-Tesla MRI scanner (Magnetom Trio; Siemens, Erlangen, Germany) with a receive-only, 8-channel, phased-array head coil and a whole-body transmitter coil. The studies at the different ages were performed in the same MRI scanner. We used foam pads on both sides of the head to minimize head motion. Two high-resolution T1-weighted image series were acquired using a magnetization-prepared, rapid acquisition gradient-echo pulse sequence (repetition time $(\mathrm{TR})=2,200 \mathrm{~ms}$; echo time $(\mathrm{TE})=3.05 \mathrm{~ms}$; inversion time $=1,100 \mathrm{~ms}$; flip angle $(\mathrm{FA})=10^{\circ}$; matrix size $=256 \times 256$; field-of-view $(\mathrm{FOV})=220 \times 220 \mathrm{~mm}$; slice thickness $=1.0 \mathrm{~mm}$ ), and proton-density and T2-weighted images were collected using a dual-echo turbo spin-echo pulse sequence $(\mathrm{TR}=8,000 \mathrm{~ms}$; TE1 and TE $2=17 \mathrm{~ms}$ and $133 \mathrm{~ms}$, respectively; $\mathrm{FA}=150^{\circ}$; matrix size $=256 \times 256$; FOV $=$ $240 \times 240 \mathrm{~mm}$; slice thickness $=5.0 \mathrm{~mm}$; turbo factor $=5$ ). After 4 years, two high-resolution T1-weighted image series were collected again using a magnetization-prepared, rapid acquisition gradient-echo pulse sequence $\left(\mathrm{TR}=2,200 \mathrm{~ms} ; \mathrm{TE}=2.34 \mathrm{~ms}\right.$; inversion time $=900 \mathrm{~ms} ; \mathrm{FA}=9^{\circ}$;

Table 1. Demographic data and biophysical variables of subjects with CCHS and control subjects

\begin{tabular}{|c|c|c|c|c|c|c|}
\hline \multirow[b]{2}{*}{ Variable } & \multicolumn{2}{|c|}{$\mathrm{CCHS}(n=7)$} & \multicolumn{2}{|c|}{ Controls $(n=3)$} & \multicolumn{2}{|c|}{$P$ value } \\
\hline & $\begin{array}{c}\text { First } \\
\operatorname{scan}(A)\end{array}$ & $\begin{array}{l}\text { Second } \\
\text { scan (B) }\end{array}$ & $\begin{array}{c}\text { First } \\
\text { scan }(C)\end{array}$ & $\begin{array}{l}\text { Second } \\
\text { scan (D) }\end{array}$ & $\begin{array}{c}\text { A vs. } \\
\text { C }\end{array}$ & $\begin{array}{c}\text { B vs. } \\
\text { D }\end{array}$ \\
\hline $\begin{array}{l}\text { Age } \\
\text { (years) }\end{array}$ & $16.1 \pm 2.7$ & $20.6 \pm 2.8$ & $15.9 \pm 2.1$ & $20.4 \pm 2.2$ & 0.92 & 0.95 \\
\hline $\begin{array}{l}\text { Gender } \\
\text { (male: } \\
\text { female) }\end{array}$ & $4: 3$ & $4: 3$ & $3: 0$ & $3: 0$ & - & - \\
\hline $\begin{array}{l}\text { BMI } \\
\left(\mathrm{kg} / \mathrm{m}^{2}\right)\end{array}$ & $19.7 \pm 4.8$ & $23.5 \pm 5.8$ & $22.6 \pm 5.9$ & $26.0 \pm 9.2$ & 0.45 & 0.61 \\
\hline
\end{tabular}

$\mathrm{CCHS}$, subjects with congenital central hypoventilation syndrome.

PHOX2B mutations: two positive; one negative; four not tested. matrix size $=320 \times 320 ; \mathrm{FOV}=230 \times 230 \mathrm{~mm}$; slice thickness $=0.9 \mathrm{~mm}$ ), and proton density- and T2-weighted images were acquired using a dual-echo, turbo spin-echo pulse sequence $(\mathrm{TR}=10,000 \mathrm{~ms}$; TE1 and $\mathrm{TE} 2=12 \mathrm{~ms}$ and $119 \mathrm{~ms}$, respectively; $\mathrm{FA}=130^{\circ}$; matrix size $=256 \times 256$; $\mathrm{FOV}=230 \times 230 \mathrm{~mm}$; slice thickness $=3.5 \mathrm{~mm}$; turbo factor $=5$ ).

\section{Data Analysis}

We examined high-resolution T1-weighted, proton density-weighted, and T2-weighted images of all subjects with CCHS and control subjects for presence of any major brain pathology, including cystic lesions, tumors, or major infarcts. No subjects with CCHS or control subjects showed any such abnormality on brain images. Highresolution T1-weighted images were also examined to confirm the absence of any head motion-related or other imaging artifacts.

The statistical parametric mapping package (SPM8; http://www.fil. ion.ucl.ac.uk/spm/), MRIcroN, and MATLAB-based (MathWorks, Natick, MA) custom software were used to process and analyze data.

\section{Realignment, Segmentation, Normalization, and Smoothing}

For each study, both high-resolution T1-weighted image volumes were realigned to remove any potential variation from head motion and averaged to increase signal-to-noise ratio. The averaged images were bias-corrected for signal intensity differences; partitioned into gray, white, and cerebrospinal fluid tissue types; and normalized to the Montreal Neurological Institute space, using the unified segmentation approach. The normalized gray matter maps were modulated (scaled to native space) and smoothed with a Gaussian filter (full width at half maximum, $10 \mathrm{~mm}$ ).

The averaged and bias-corrected T1-weighted images from individual subjects with CCHS and control subjects were also normalized to Montreal Neurological Institute space. The normalized images of all subjects with CCHS and controls were averaged to create background images for structural identification.

\section{Statistical Analysis}

The normalized and smoothed gray matter maps of control subjects were compared voxel by voxel between the two time points using paired $t$-tests (uncorrected threshold, $P=0.001$; minimum extended cluster size, 5 voxels). Similarly, the normalized and smoothed gray matter maps of subjects with CCHS were also compared between first and second time points using paired $t$-tests (uncorrected threshold, $P=0.001$; minimum extended cluster size, 5 voxels). The clusters showing significantly reduced gray matter volume across the brain in control subjects were converted into a brain mask, and used to exclude brain sites showing reduced gray matter volume from subjects with CCHS for partitioning normal age-related changes. The statistical parametric maps with clusters showing significant differences between two time points in subjects with CCHS, corrected for normal age-related changes, were overlaid onto background images for structural identification.

\section{ACKNOWLEDGMENTS}

We thank Rebecca Harper, Edwin Valladares, and Annaise Magliore for assistance with data collection; the parents and children for participating in this research; and the family of Evan Tessier for their support.

\section{STATEMENT OF FINANCIAL SUPPORT}

This study was supported by National Institute of Child Health and Human Development grant no. R01 HD-22695.

\section{REFERENCES}

1. Dauger S, Pattyn A, Lofaso F, et al. Phox $2 \mathrm{~b}$ controls the development of peripheral chemoreceptors and afferent visceral pathways. Development 2003;130:6635-42.

2. American Thoracic Society. Idiopathic congenital central hypoventilation syndrome: diagnosis and management. Am J Respir Crit Care Med 1999;160:368-73.

3. Ruof H, Hammer J, Tillmann B, Ghelfi D, Weber P. Neuropsychological, behavioral, and adaptive functioning of Swiss children with congenital central hypoventilation syndrome. J Child Neurol 2008;23:1254-9. 
4. Vanderlaan M, Holbrook CR, Wang M, Tuell A, Gozal D. Epidemiologic survey of 196 patients with congenital central hypoventilation syndrome. Pediatr Pulmonol 2004;37:217-29.

5. Kumar R, Lee K, Macey PM, Woo MA, Harper RM. Mammillary body and fornix injury in congenital central hypoventilation syndrome. Pediatr Res 2009;66:429-34.

6. Kumar R, Macey PM, Woo MA, Alger JR, Harper RM. Elevated mean diffusivity in widespread brain regions in congenital central hypoventilation syndrome. J Magn Reson Imaging 2006;24:1252-8.

7. Kumar R, Macey PM, Woo MA, Alger JR, Harper RM. Diffusion tensor imaging demonstrates brainstem and cerebellar abnormalities in congenital central hypoventilation syndrome. Pediatr Res 2008;64:275-80.

8. Kumar R, Macey PM, Woo MA, Alger JR, Keens TG, Harper RM. Neuroanatomic deficits in congenital central hypoventilation syndrome. J Comp Neurol 2005;487:361-71.

9. Gozal D, Daniel JM, Dohanich GP. Behavioral and anatomical correlates of chronic episodic hypoxia during sleep in the rat. J Neurosci 2001;21:2442-50.

10. Veasey SC, Davis CW, Fenik P, et al. Long-term intermittent hypoxia in mice: protracted hypersomnolence with oxidative injury to sleep-wake brain regions. Sleep 2004;27:194-201.

11. Cai J, Tuong CM, Zhang Y, et al. Mouse intermittent hypoxia mimicking apnoea of prematurity: effects on myelinogenesis and axonal maturation. J Pathol 2012;226:495-508.

12. Cai J, Tuong CM, Gozal D. A neonatal mouse model of intermittent hypoxia associated with features of apnea in premature infants. Respir Physiol Neurobiol 2011;178:210-7.

13. Soria G, Aguilar E, Tudela R, Mullol J, Planas AM, Marin C. In vivo magnetic resonance imaging characterization of bilateral structural changes in experimental Parkinson's disease: a T2 relaxometry study combined with longitudinal diffusion tensor imaging and manganese-enhanced magnetic resonance imaging in the 6-hydroxydopamine rat model. Eur J Neurosci 2011;33:1551-60.

14. Segall JM, Turner JA, van Erp TG, et al. Voxel-based morphometric multisite collaborative study on schizophrenia. Schizophr Bull 2009;35:82-95.

15. Good CD, Johnsrude IS, Ashburner J, Henson RN, Friston KJ, Frackowiak RS. A voxel-based morphometric study of ageing in 465 normal adult human brains. Neuroimage 2001;14:21-36.

16. Gogtay N, Giedd JN, Lusk L, et al. Dynamic mapping of human cortical development during childhood through early adulthood. Proc Natl Acad Sci USA 2004;101:8174-9.

17. Alexander GE, Chen K, Merkley TL, et al. Regional network of magnetic resonance imaging gray matter volume in healthy aging. Neuroreport 2006;17:951-6.

18. Kumar R, Delshad S, Macey PM, Woo MA, Harper RM. Development of T2-relaxation values in regional brain sites during adolescence. Magn Reson Imaging 2011;29:185-93.

19. Kumar R, Nguyen HD, Macey PM, Woo MA, Harper RM. Regional brain axial and radial diffusivity changes during development. J Neurosci Res 2012;90:346-55.

20. Kumar R, Delshad S, Woo MA, Macey PM, Harper RM. Age-related regional brain T2-relaxation changes in healthy adults. J Magn Reson Imaging 2012;35:300-8.

21. Miura M, Reis DJ. Cerebellum: a pressor response elicited from the fastigial nucleus and its efferent pathway in brainstem. Brain Res 1969;13:595-9.
22. Lutherer LO, Lutherer BC, Dormer KJ, Janssen HF, Barnes CD. Bilateral lesions of the fastigial nucleus prevent the recovery of blood pressure following hypotension induced by hemorrhage or administration of endotoxin. Brain Res 1983;269:251-7.

23. Oppenheimer SM, Wilson JX, Guiraudon C, Cechetto DF. Insular cortex stimulation produces lethal cardiac arrhythmias: a mechanism of sudden death? Brain Res 1991;550:115-21.

24. Coote JH. A role for the paraventricular nucleus of the hypothalamus in the autonomic control of heart and kidney. Exp Physiol 2005;90:169-73.

25. Dampney RA, Coleman MJ, Fontes MA, et al. Central mechanisms underlying short- and long-term regulation of the cardiovascular system. Clin Exp Pharmacol Physiol 2002;29:261-8.

26. Oppenheimer SM, Cechetto DF, Hachinski VC. Cerebrogenic cardiac arrhythmias. Cerebral electrocardiographic influences and their role in sudden death. Arch Neurol 1990;47:513-9.

27. Cechetto DF, Chen SJ. Subcortical sites mediating sympathetic responses from insular cortex in rats. Am J Physiol 1990;258:R245-55.

28. Oppenheimer SM, Hachinski VC. The cardiac consequences of stroke. Neurol Clin 1992;10:167-76.

29. Woo MS, Woo MA, Gozal D, Jansen MT, Keens TG, Harper RM. Heart rate variability in congenital central hypoventilation syndrome. Pediatr Res 1992;31:291-6.

30. Trang H, Boureghda S, Denjoy I, Alia M, Kabaker M. 24-hour BP in children with congenital central hypoventilation syndrome. Chest 2003;124:1393-9.

31. Silvestri JM, Hanna BD, Volgman AS, Jones PJ, Barnes SD, Weese-Mayer DE. Cardiac rhythm disturbances among children with idiopathic congenital central hypoventilation syndrome. Pediatr Pulmonol 2000;29: 351-8.

32. Gronli JO, Santucci BA, Leurgans SE, Berry-Kravis EM, Weese-Mayer DE. Congenital central hypoventilation syndrome: PHOX2B genotype determines risk for sudden death. Pediatr Pulmonol 2008;43:77-86.

33. Mendez MF, Adams NL, Lewandowski KS. Neurobehavioral changes associated with caudate lesions. Neurology 1989;39:349-54.

34. Poldrack RA, Prabhakaran V, Seger CA, Gabrieli JD. Striatal activation during acquisition of a cognitive skill. Neuropsychology 1999;13:564-74.

35. Lombardi WJ, Andreason PJ, Sirocco KY, et al. Wisconsin Card Sorting Test performance following head injury: dorsolateral fronto-striatal circuit activity predicts perseveration. J Clin Exp Neuropsychol 1999;21:2-16.

36. Arnsten AF, Li BM. Neurobiology of executive functions: catecholamine influences on prefrontal cortical functions. Biol Psychiatry 2005;57:1377-84.

37. Zhang X, Ma L, Li S, Wang Y, Wang L. A functional MRI evaluation of frontal dysfunction in patients with severe obstructive sleep apnea. Sleep Med 2011;12:335-40.

38. Newman LM, Trivedi MA, Bendlin BB, Ries ML, Johnson SC. The Relationship Between Gray Matter Morphometry and Neuropsychological Performance in a Large Sample of Cognitively Healthy Adults. Brain Imaging Behav 2007;1:3-10.

39. Goldberg DS, Ludwig IH. Congenital central hypoventilation syndrome: ocular findings in 37 children. J Pediatr Ophthalmol Strabismus 1996;33:175-80.

40. Kumar R, Nguyen HD, Macey PM, Woo MA, Harper RM. Dilated basilar arteries in patients with congenital central hypoventilation syndrome. Neurosci Lett 2009;467:139-43. 